\title{
Covid-19: proportionnalité des incitations à la vaccination
}

\section{Kaspar Gerber}

Dr en droit, LL.M., collaborateur scientifique (postdoc), chaire de droit public, droit administratif et droit des assurances sociales, Université de Zurich

L'Etat est autorisé à inciter la population à se faire vacciner si les moyens prévus sont proportionnés. Le présent article montre quand les informations, les recommandations, l'obligation vaccinale et le certificat Covid satisfont au principe de proportionnalité. Les explications reposent sur la situation juridique telle qu'elle a été exposée dans le numéro 47.

Si l'Etat veut encourager la population à se faire vacciner dans le cas du Covid-19 (mais pas seulement), il peut prendre des mesures dans ce sens. Il faut toutefois qu'elles respectent la proportionnalité, c.-à-d. qu'elles cumulent les conditions suivantes: être aptes, nécessaires et "raisonnablement exigibles» pour atteindre un objectif étatique. "Raisonnablement exigible» signifie qu'il doit y avoir une relation raisonnable entre les fins et les moyens [1]. Lorsque l'efficacité et la sécurité des vaccins concernés sont données, deux facteurs sont décisifs pour contrôler la proportionnalité de mesures étatiques d'incitation à la vaccination: le type ou l'intensité des mesures étatiques et leurs groupes cibles. L'Etat dispose de plusieurs mesures offrant un degré d'intensité différent: les informations, les recommandations, l'obligation vaccinale et le certificat Covid-19.

\section{Informations}

En vertu de la Loi sur les épidémies (LEp), l'Office fédéral de la santé publique (OFSP) informe des risques que présentent les maladies transmissibles et des mesures possibles pour les prévenir et les combattre. Ces informations sont destinées au grand public, aux professionnels de la santé, à certains groupes de personnes (notamment si elles sont particulièrement concernées) et aux autorités (également en-dehors du domaine de la santé) [2].

Informer des risques de maladie ou promouvoir la santé par des campagnes d'information sont des éléments clés de la protection de la santé publique. Les citoyennes et citoyens devraient prendre leurs décisions autant que possible sur la base d'informations appropriées. Comme les décisions et comportements person- nels peuvent avoir un impact sur sa propre santé et, en particulier, sur celle d'autrui, il faut que l'Etat mette en place des options d'action et des bases de décision [3].

\section{Orientation sur des groupes cibles}

L'Etat informe la population à différents niveaux, en adaptant les méthodes et les instruments en fonction des objectifs poursuivis. Au niveau individuel par exemple, il peut mettre en place un service de conseil et des informations aux personnes exposées à un risque particulier. Au niveau de certains groupes de personnes particulièrement vulnérables, p. ex. les migrantes et les migrants, il veillera à les informer de manière ciblée. Cela permet de réduire la mise en danger de tiers, p. ex. d'autres membres de la famille, et de mettre l'accent sur les intérêts sanitaires des individus auxquels on s'adresse (p. ex. brochures de vaccinations pour parents d'enfants en bas âge) [4].

Le critère d'admissibilité d'une recommendation est déterminé par le principe de proportionnalité.

Les informations diffusées par l'Etat doivent reposer sur les principes généraux de l'administration: les autorités sont tenues d'informer de manière compréhensible, objective, transparente et conforme au principe de proportionnalité [5]. Les canaux envisageables sont les communiqués et conférences de presse, la mise à disposition ou remise de documents de sensibilisation, les informations sur internet ou via une hotline, le bulletin de l'OFSP en tant que revue spécialisée officielle [6], et les campagnes d'information dans les médias, comme ce fut déjà le cas lors de la campagne Stop Sida; 


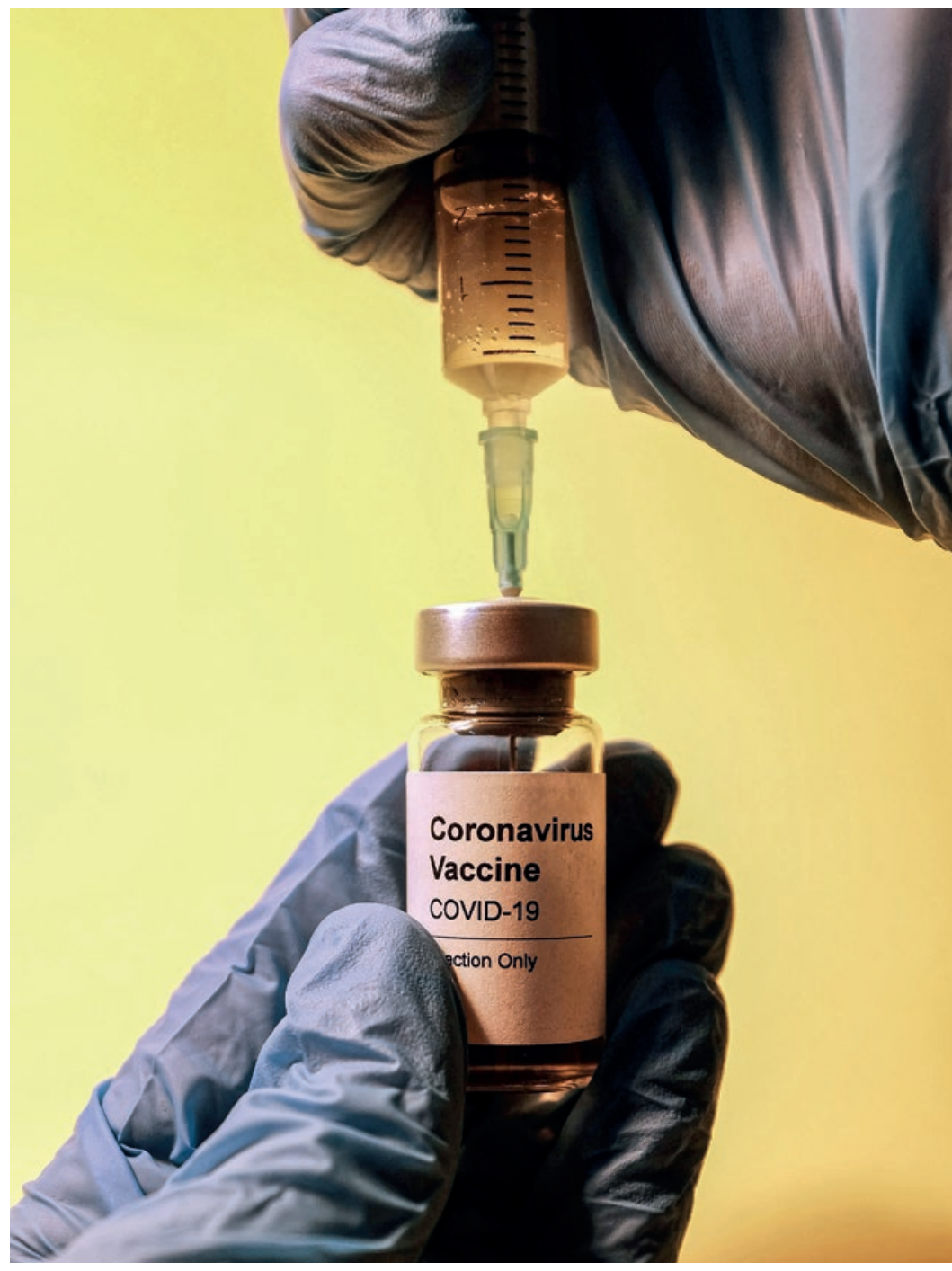

sans oublier les supports publicitaires en tous genres, p. ex. affiches ou flyers dans les lieux accessibles au public [7].

\section{Communication coordonnée}

Pour garantir une information cohérente et suivre une même ligne, il est indispensable que l'OFSP et les cantons se coordonnent suffisamment dans le cadre de leurs compétences. Ils tiennent tout particulièrement compte des objectifs et stratégies nationaux. L'OFSP et les autorités cantonales sont d'ailleurs tenus par la loi de coordonner leurs activités d'information [8]. L'OFSP met à la disposition des professionnels de la santé des renseignements généraux sous forme de recommandations, de directives et d'informations établies conjointement avec des partenaires et portant sur la vaccination, les vaccins et les soins prophylactiques. Leur but est de protéger les individus et la population dans son ensemble [9]. En revanche, les informations de l'OFSP ne remplaceront jamais l'entretien individuel entre un médecin et son patient (informed consent).

\section{Informations sur le Covid-19}

L'OFSP met à disposition une documentation complète sur le Covid-19, incluant notamment plusieurs explications médicales et des indications utiles pour les situations quotidiennes [10]. Il a par ailleurs élaboré un grand nombre d'informations destinées explicitement aux professionnels de la santé sur les marches à suivre face au Covid-19 [11].

\section{Recommandations}

L'OFSP publie aussi des recommandations sur les mesures de lutte contre les maladies transmissibles et les agents pathogènes et adapte régulièrement ces recommandations à l'état des connaissances scientifiques. Si d'autres offices fédéraux sont concernés, l'OFSP agit avec leur accord [12].

Ces recommandations contiennent des instructions sur les comportements à adopter. Elles s'adressent à l'ensemble de la population, à des groupes de personnes particulièrement vulnérables ou à certains groupes cibles. Le critère d'admissibilité d'une recommandation est déterminé par le principe de proportionnalité, selon lequel une raison suffisante doit justifier la formulation d'une recommandation. C'est le cas lorsqu'un bien juridiquement protégé est exposé à un risque [13] et, dans le cas des maladies transmissibles, ces biens juridiquement protégés sont la santé individuelle ou publique, mais aussi le bon fonctionnement socio-économique de la société qui va de pair avec la santé.

\section{Règle de l'objectivité}

Lorsque l'Etat publie des recommandations, il n'a nul besoin de se limiter à la communication de faits mais peut aussi en tirer des conclusions selon sa propre appréciation dans le but de guider avec efficacité le comportement du grand public. De manière générale, les recommandations étatiques sont soumises à la règle de l'objectivité [14].

Les plans d'urgence spécifiques à une maladie (p. ex. le plan suisse de pandémie Influenza [15]) sont aussi considérés comme des recommandations. En cas de lutte contre les maladies infectieuses, il se peut que d'autres offices fédéraux soient concernés, comme l'Office fédéral de l'environnement (OFEV), p. ex. dans la lutte contre des insectes, ou l'Office fédéral de la sécu- 
rité alimentaire et des affaires vétérinaires (OSAV) dans le cas des zoonoses. Pour que les mesures s'appliquent avec succès, il est impératif que les recommandations soient mises en place en concertation avec les services concernés [16].

\section{Caractère contraignant des recomman- dations}

Aussi pertinentes soient-elles, les recommandations de l'OFSP peuvent être ou ne pas être suivies. Ne pas les respecter, comme ne pas se tenir aux mesures d'hygiène obligatoires (p. ex. le port du masque) ou aux restrictions d'accès à des lieux publics, ne peut avoir de conséquence directe sur les droits fondamentaux des personnes concernées. Il n'existe aucune base légale dans ce sens. En revanche, il se peut qu'une recommandation, lorsqu'elle est suivie, engendre un préjudice (financier) à des particuliers et engage, de ce fait, la responsabilité de l'Etat [17]. Les recommandations de l'OFSP ne changent rien à la nécessité de l'entretien individuel entre le médecin et le patient (informed consent).

\section{Recommandations de vaccination contre le Covid-19}

Les recommandations de vaccination contre le Covid-19, tout comme les recommandations de vaccination pour d'autres maladies transmissibles, sont rédigées par la Commission fédérale pour les vaccinations (CFV) en collaboration avec l'OFSP [18]. Dès que Swissmedic accorde une autorisation de mise sur le marché à un vaccin, la CFV élabore des recommandations en se basant sur les résultats des «essais cliniques de phase III». Les dernières découvertes scientifiques et la situation épidémiologique actuelle sont prises en compte dans les recommandations de vaccination [19]. Lors du contrôle de la proportionnalité des recommandations, une question clé se pose: à quel point les groupes de personnes sont-ils touchés par le Covid-19? Les groupes à risque ont été définis peu après le début de la pandémie, donc il y a un an et demi, dans les premières ordonnances Covid-19 [20]. Selon l'appréciation actuelle, le coronavirus peut être dangereux pour les personnes âgées, les femmes enceintes, les adultes avec trisomie 21 ou certaines formes de maladies chroniques [21]. Au fil de l'expérience acquise, le profil de risque n'a cessé d'être précisé. La stratégie actuelle de vaccination contre le Covid-19 distingue six groupes cibles selon l'âge, l'état de santé et le type d'activité [22]. Les recommandations émises sont extrêmement détaillées et tiennent compte de ces groupes de personnes exposés au risque de manière différente [23]. Lorsque, du point de vue épidémiologique, le taux de vaccination est faible dans certains groupes de population, les efforts d'information des autorités doivent se concentrer sur eux selon une approche basée sur le risque. C'est ce que dicte le principe de proportionnalité.

\section{L'obligation vaccinale}

Dans certaines circonstances, le non-respect des recommandations des autorités par le grand public peut devenir un problème socio-médical. Dans de tels cas, l'obligation vaccinale peut être envisagée en tant qu'ultima ratio, p. ex. pour maintenir l'activité de certains services dans les hôpitaux ou les homes [24]. Le contrôle de la proportionnalité d'une telle obligation exige de soupeser avec soin différents facteurs qui sont, en priorité, la situation de menace concrète, les

Ne pas respecter les recommendations, ne peut pas avoir de conséquence directe sur les droits fondamentaux des personnes concernées.

avantages épidémiologiques attendus de l'obligation vaccinale, et la baisse de motivation au travail des personnes soumises à cette obligation.

\section{Le certificat Covid-19}

Depuis le 13 septembre 2021, le certificat Covid-19 est obligatoire à l'intérieur des restaurants, des lieux de culture et de loisirs pour les personnes de plus de 16 ans, ainsi que lors de manifestations se déroulant à l'intérieur [25]. Le certificat est établi pour les personnes vaccinées, guéries ou testées. Depuis le 11 octobre 2021, en plus de l'obligation de certificat, la Confédération ne prend plus en charge les coûts de participation individuelle à un test permettant d'obtenir un certificat, hormis certaines exceptions pour les personnes qui ont obtenu leur première dose de vaccin et les jeunes [26].

De ce fait, l'option de "personnes testées» est rendue nettement plus difficile. Dans certains cas, cela peut générer une pression considérable sur la vaccination contre le Covid-19. Le certificat Covid-19 se trouve en quelque sorte pris entre deux fronts (la recommandation et l'obligation vaccinales). Les questions juridiques à ce propos ne sont pas encore complètement clarifiées.

Crédits photo

Hakan Nural / Unsplash

\section{Références}

La liste complète des références est disponible dans la version en ligne de l'article sur www.bullmed.ch 